\title{
The Relationship of the Post-reflux Swallow-induced Peristaltic Wave Index and Esophageal Baseline Impedance with Gastroesophageal Reflux Disease Symptoms
}

\author{
Young Kyu Cho, ${ }^{1}$ Joon Seong Lee, ${ }^{1 *}$ Tae Hee Lee, ${ }^{1}$ Su Jin Hong, ${ }^{2}$ Sang Joon Park, ${ }^{1}$ Seong Ran Jeon, ${ }^{1}$ Hyun Gun Kim, ${ }^{1}$ and Jin-Oh Kim ${ }^{1}$ \\ ${ }^{I}$ Department of Internal Medicine, College of Medicine, Soonchunhyang University Hospital, Seoul, Korea; and ${ }^{2}$ Department of Internal \\ Medicine, Soonchunhyang University Hospital, Bucheon, Gyeonggi-do, Korea
}

\section{Background/Aims}

The post-reflux swallow-induced peristaltic wave (PSPW) index and esophageal baseline impedance (BI) are novel impedance parameters used to evaluate esophageal chemical clearance and mucosal integrity. However, their relationship with reflux symptoms is not known. We aim to evaluate the correlations of PSPW index and esophageal BI with gastroesophageal reflux disease (GERD) symptoms.

\section{Methods}

We performed a retrospective review of multichannel intraluminal impedance and $\mathrm{pH}(\mathrm{MII}-\mathrm{pH})$ tracings in patients with suspected GERD. Reflux symptoms were also analyzed from checklists using ordinal scales. The PSPW index and esophageal Bls in 6 spots (z1-z6) were measured. Bivariate (Spearman) correlation was used to analyze the relationship between the PSPW index or esophageal BI, and the degree of GERD symptoms measured.

\section{Results}

The MII-pH records of 143 patients were analyzed. The PSPW index was significantly lower in patients who had heartburn and negatively correlated with the degree of heartburn $(r=-0.186, P<0.05)$. On the contrary, the PSPW index was not significantly correlated with the degree of dysphagia $(r=-0.013, P=0.874)$. Distal esophageal BI was not significantly correlated with heartburn, but negatively correlated with the degree of dysphagia (z3: $r=-0.328, z 4: r=-0.361, z 5: r=-0.316, z 6: r=-0.273 ; P<0.05)$.

\section{Conclusions}

These findings suggest that delayed chemical clearance of the esophagus may induce heartburn, but that it is not related to dysphagia. However, a lack of esophageal mucosal integrity may be related to dysphagia.

(J Neurogastroenterol Motil 2017;23:237-244)

Key Words

Dysphagia; Esophageal baseline impedance; Gastroesophageal reflux; Heartburn; PSPW index

Received: July 15, 2016 Revised: July 18, 2016 Accepted: October 31, 2016

(a) This is an Open Access article distributed under the terms of the Creative Commons Attribution Non-Commercial License (http://creativecommons. org/licenses/by-nc/4.0) which permits unrestricted non-commercial use, distribution, and reproduction in any medium, provided the original work is properly cited.

*Correspondence: Joon Seong Lee, MD Institute for Digestive Research, Soonchunhyang University College of Medicine, 59, Daesagwan-ro, Yongsan-gu, Seoul 04401, Korea

Tel: +82-2-709-9691, Fax: +82-2-709-9696, E-mail: joonlee@schmc.ac.kr 


\section{Introduction}

Gastroesophageal reflux disease (GERD) is currently defined as a condition in which refluxed gastric contents in the esophagus causes troublesome symptoms and/or complications. ${ }^{1}$ Although many patients with GERD manifest typical symptoms such as heartburn and acid regurgitation, others predominantly complain of atypical symptoms, such as hoarseness, throat clearing, burning throat, water brash, halitosis, chest pain, chronic cough, dysphagia, and dental erosion. ${ }^{2}$ Multichannel intraluminal impedance and $\mathrm{pH}$ (MII-pH) monitoring can be used to confirm GERD and it has diagnostic gain compared with using a $\mathrm{pH}$-meter alone for patients with refractory heartburn treated with proton pump inhibitors (PPIs). ${ }^{3}$ It can detect the movement of esophageal contents during peristalsis or reflux, and discriminate the acidity and nature of the contents (liquid, gas, or mixed). Once reflux occurs, the distended esophageal wall by refluxed gastric material activate the stretch receptors in the esophageal wall, and induce the secondary peristalsis which forces the refluxed bolus quickly back to the stomach. ${ }^{4} \mathrm{An}$ other defense mechanism is primary peristalsis known as the postreflux swallow-induced peristaltic wave (PSPW). PSPW index has been confirmed to improve the diagnostic efficacy of MII-pH monitoring. One study showed that the PSPW index was significantly lower in patients with erosive reflux disease (ERD) and nonerosive reflux disease (NERD) than in patients with functional heartburn, regardless of PPI therapy or anti-reflux surgery. ${ }^{5}$

Esophageal intraluminal baseline impedance (BI) may represent the status of mucosal integrity. ${ }^{6}$ Farre et $\mathrm{al}^{7}$ showed that the esophageal BI decreases to and maintains a low value after acid perfusion in their acid perfusion study. They demonstrated that esophageal BI measurements can be used to evaluate changes in the esophageal mucosa integrity after acid perfusion injury, and showed that it is correlated with transepithelial resistance (TER), which reflects impaired mucosal structure. Dilated intercellular space (DIS) in the esophageal squamous epithelium is generally regarded as a structural marker of GERD and potentially as an early injury of esophageal mucosal integrity. ${ }^{8}$ DIS occurs in association with a decrease in TER. ${ }^{9}$ Photomicrographs of intercellular spaces in patients with reflux esophagitis and NERD showed more dilation compared with that of controls. ${ }^{6}$ Moreover, esophageal BI values were lower in GERD patients than in controls and were lower in reflux esophagitis patients than in NERD patients. ${ }^{6}$

While GERD patients have a lower PSPW index and esophageal BI than those of controls, no study has directly compared
GERD symptoms with the PSPW index or esophageal BI. The aim of this study is to evaluate the correlations of the PSPW index and esophageal BI with GERD symptoms.

\section{Materials and Methods}

We retrospectively selected patients with suspected GERD symptoms who underwent 24-hour ambulatory MII-pH monitoring between May 2007 and December 2011. Inclusion criteria were as follows: age $>19$ years, completion of the GERD symptom questionnaire, and a MII-pH monitoring duration of at least 21 hours. The patients' symptoms were assessed using a validated questionnaire completed prior to MII-pH monitoring. Exclusion criteria were study after endoscopic manipulation such as Stretta therapy, after fundoplication, previous esophageal or gastric endoscopic treatment such as polypectomy, endoscopic mucosal resection, endoscopic submucosal dissection, balloon or bougie dilation, previous esophagogastric surgery such as esophageal resection, gastric wedge resection, gastrectomy, and study on PPI. Ineffective esophageal motility by esophageal manometry was included in the study as it is a common phenomenon of GERD. All patients underwent upper gastrointestinal endoscopy and most of them showed normal findings (only 3\% showed reflux esophagitis of Los Angeles classification A). This study was approved by the Institutional Review Board of the Soonchunhyang University Seoul Hospital.

\section{Multichannel Intraluminal Impedance and pH Monitoring Parameters: The Post-reflux Swallow-induced Peristaltic Wave Index and Esophageal Baseline Impedance}

All subjects underwent MII-pH monitoring (Sandhill Scientific, Inc, Highland Ranch, CO, USA) after PPI withdrawal for at least 7 days. The $\mathrm{pH}$ electrode was placed $5 \mathrm{~cm}$ above the upper margin of the manometrically defined lower esophageal sphincter (LES), and $6 \mathrm{BI}$ values (z1, z2, z3, z4, z5, and z6) were determined at 6 sites $(3,5,7,9,15$, and $17 \mathrm{~cm}$ above the LES respectively). The subjects were encouraged to maintain their normal daily activities and diet. Event markers on the data logger were used to record the start and end times of symptoms, meal times, and position changes. Impedance, $\mathrm{pH}$, and symptom signals were collected at a sampling rate resolution of $50 \mathrm{~Hz}$. Recorded data were analyzed using a dedicated software program (BioView Analysis; Sandhill Scientific, Inc) in conjunction with a visual analysis using appropriate time window and zooming. A PSPW was defined as an antegrade $50 \%$ drop in impedance level relative to the baseline (bolus 
entry) originating in the most proximal site of impedance, reaching all distal impedance sites, and followed by at least a $50 \%$ return to baseline at all distal impedance sites (bolus exit) (Fig. 1). ${ }^{10}$ Postreflux swallows not fulfilling this criteria were excluded. To exclude the spontaneous swallowing of around 64 swallows per hour ${ }^{11}$ only a PSPW occurring within 30 seconds from the end of reflux episodes was taken into account considering the 10 to 15 seconds of salivary gland response after esophageal acidification. ${ }^{12}$ For each impedance$\mathrm{pH}$ monitoring tracing, the number of PSPWs was calculated and divided by the total reflux events to obtain the PSPW index, a parameter representing the efficacy of chemical clearance. These manual processes were performed by one investigator (first author). Esophageal BI was calculated using a software program (BioView Analysis, BL Plot program V2.0; Sandhill Scientific, Inc). We evaluated the associations of the PSPW index and esophageal BI with GERD symptoms.

All subjects also underwent high-resolution esophageal manometry (Given Imaging, Los Angeles, CA, USA) just before MII-pH. We measured 10 times the $10 \mathrm{~mL}$ water swallow using distilled water with intervals of at least 20 seconds. The recorded data were analyzed after thermal compensation using a dedicated

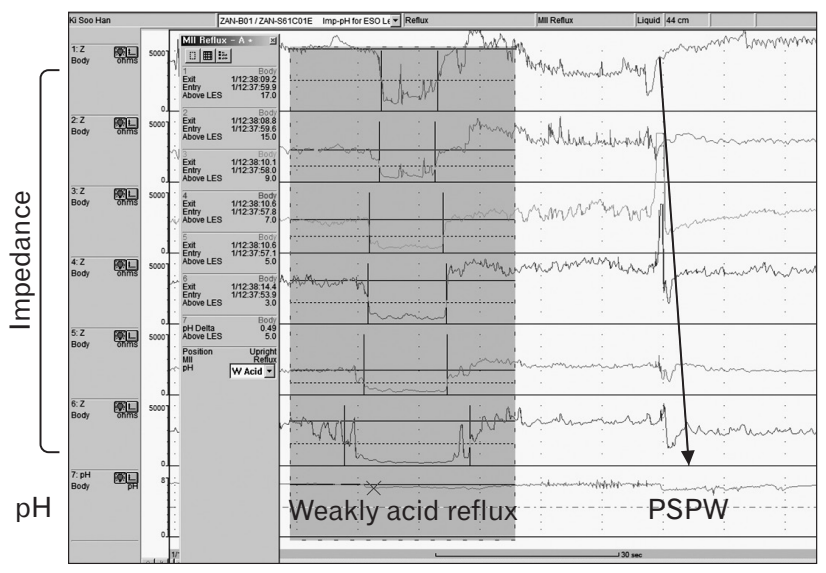

Figure 1. Post-reflux swallow-induced peristaltic wave (PSPW) in multichannel intraluminal impedance and $\mathrm{pH}$ monitoring. Upper 6 pannel means impedance from upper to lower esophagus (Z1 to Z6: 3, $5,7,9,15$, and $17 \mathrm{~cm}$ above the lower esophageal sphincter [LES]), the lowest pannel means $\mathrm{pH}$ in the distal esophagus at $5 \mathrm{~cm}$ from the upper margin of LES. Gray box means reflux event (weakly acid and liquid reflux in this case) and each bars in impedance graph suggest entry and exit of materials. Once reflux occurs, the bulk of refluxed materials are quickly expelled from the esophageal lumen to the stomach by a secondary peristaltic wave. This secondary peristalsis is called PSPW; arrow) and it is local reflex response elicited by stretch receptors in the esophagus. software (Manoview; Given Imaging). The Chicago classification version 3.0 was applied to disease category.

\section{Classification of Reflux Episodes According to Acidity, Post-reflux Swallow-induced Peristaltic Wave, and Neutralization}

We classified reflux events into five categories according to the acidity of the intraluminal esophagus when reflux and PSPWs occurred. When the $\mathrm{pH}$ of a reflux event was lower than 4 , it was termed "acid reflux," while "non-acid reflux" referred to reflux events with a $\mathrm{pH}$ above 4. After a PSPW occurred during acid reflux, some cases showed neutralization, which seemed to be due to the PSPW, but other cases still showed acidity. The following categories were used: (1) acid reflux not followed by PSPW, (2) acid reflux followed by a PSPW with neutralization, (3) acid reflux followed by PSPW without neutralization, (4) non-acid reflux not followed by a PSPW, and (5) non-acid reflux followed by a PSPW. The correlation between each category and GERD symptoms was analyzed.

\section{Gastroesophageal Reflux Disease Symptom Scores}

Symptoms including dysphagia, heartburn, acid regurgitation, globus sense, and chest pain were scored by frequency and severity, each on a Likert scale ranging from 0 to 4 . We asked patients to rate the frequency of their symptoms as follows: 0 , no symptoms; 1 , less than once per month; 2, 2 to 4 times per month; 3, once to 6 times per week; and 4, daily. The patients also rated the severity of their symptoms as follows: 0 , no symptoms; 1 , can be ignored with effort; 2, cannot be ignored but does not influence one's daily activities; 3 , cannot be ignored and limits one's concentration on daily activities; and 4, cannot be ignored and markedly limits one's daily activities and often requiring rest. Symptom scores were calculated by summing the frequency and severity scores. These symptoms were evaluated just before insertion of the MII-pH monitoring catheter.

\section{Statistical Methods}

Spearman rank correlation coefficients were used to analyze the correlations of all continuous variables including symptoms with the PSPW index and esophageal BI. A $P$-value $<0.05$ was considered to indicate statistical significance. All statistical analyses were performed using SPSS software (SPSS version 18.0; IBM Corp, Armonk, NY, USA). 


\section{Results}

\section{Patient Characteristics}

A total of 143 patients (60 males, mean age 58 years, range 2685 years) were enrolled in this study, and their MII-pH records were analyzed (Table 1). Indications for performing MII-pH monitoring were as follows: heartburn $(n=25)$, acid regurgitation $(\mathrm{n}=55)$, chest pain $(\mathrm{n}=25)$, dysphagia $(\mathrm{n}=24)$, cough $(\mathrm{n}$ $=36)$, hoarseness $(\mathrm{n}=34)$, and other symptoms such as globus sense $(n=69)$. The mean symptom scale scores for heartburn, acid regurgitation, and dysphagia were 6.20, 5.03, and 5.95, respectively (Table 1).

\section{Correlations Between the Post-reflux Swallow-induced Peristaltic Wave Index and Gastroesophageal Reflux Disease Symptoms}

The PSPW index ranged from 0.00 to 0.71 with a median score of 0.13 . The PSPW index negatively and weakly correlated with the degree of heartburn $(r=-0.186, P<0.05$; Fig. 2A). However, it was not significantly correlated with the degree of dysphagia ( $P=0.874$; Fig. $2 \mathrm{~B})$. Other symptoms such as acid regurgitation, chest pain, cough, hoarseness and globus sense were not significantly correlated with the PSPW index (Fig. 2C and 2D).

\section{Correlations Between Reflux Categories and Gastroesophageal Reflux Disease Symptoms}

Mean total number of reflux event was 30.9 and the mean number of each categories were 13.8 in acid reflux not followed by

Table 1. Patient Baseline Characteristics ( $\mathrm{N}=143)$

\begin{tabular}{ll}
\hline \multicolumn{1}{c}{ Characteristics } \\
\hline Age (mean age [range], yr) & $58(26-85)$ \\
Gender (n) & 60 \\
Male & 83 \\
Female & \\
Symptoms (n) & 24 \\
Dysphagia & 25 \\
Heartburn & 55 \\
Acid regurgitation & 36 \\
Cough & 34 \\
Hoarseness & 25 \\
Chest pain & 69 \\
Others (globus sense, belching, etc) & \\
\hline
\end{tabular}

PSPW, 1.8 in acid reflux followed by a PSPW with neutralization, 1.0 in acid reflux followed by PSPW without neutralization, 12.9 in non-acid reflux not followed by a PSPW, and 1.4 in non-acid reflux followed by a PSPW (Table 2). No reflux category was significantly correlated with GERD symptoms. Distal esophageal BI $(3 \mathrm{~cm}$ and $5 \mathrm{~cm}$ above the LES) was significantly but weakly correlated with acid reflux without a PSPW $(r=-0.267$ and $r=-0.283$ respectively, $P=0.001$; Supplement Fig. 1).

\section{Correlations Between Esophageal Baseline Impedance and Gastroesophageal Reflux Disease Symptoms}

Distal esophageal BI levels of $3 \mathrm{~cm}(r=-0.273, P<0.05)$, $5 \mathrm{~cm}(r=-0.316, P<0.05), 7 \mathrm{~cm}(r=-0.361, P<0.05)$, and $9 \mathrm{~cm}(r=-0.328, P<0.05)$ above the LES were negatively and weakly associated with dysphagia scores. However, the proximal esophageal BI levels of $15 \mathrm{~cm}(P=0.236)$ and $17 \mathrm{~cm}(P=0.366)$ above the LES were not significantly correlated with dysphagia scores (Fig. 3A). Moreover, esophageal BI and heartburn were not correlated (Fig. 3B). The BI of the proximal esophagus $(17 \mathrm{~cm}$ and $15 \mathrm{~cm}$ above the LES) and of the distal esophagus ( $5 \mathrm{~cm}$ above the LES) were weakly but positively associated with acid regurgitation. Chest pain $(P<0.05)$ and hoarseness $(P<0.05)$ were positively and weakly correlated with esophageal BI only at a level of $9 \mathrm{~cm}$ above the LES (Supplementary Fig. 2).

\section{Discussion}

The pathophysiological mechanisms underlying heartburn and other GERD symptoms are poorly understood ${ }^{13}$; indeed, several mechanisms of their development have been proposed. GERD symptoms may be triggered by increased reflux, or by an increased perception of physiological reflux ${ }^{14}$ and can result from the direct effects of stimuli such as acid, bile, pepsin and temperature. An increased volume of reflux events may lead to esophageal wall distension, which triggers stretch receptors, and induces symptoms. ${ }^{15}$ Other mechanisms include motor reactions such as hypermotility and sustained longitudinal muscle contraction, as well as modulation by visceral hypersensitivity and cultural-psychological and extraesophageal factors. ${ }^{16}$ Chemical stimulation is likely related to increased permeability of the esophageal squamous epithelium, which reflect impaired tertiary defense mechanism, resulting in mucosal DIS that facilitates contact between acid or other components and sensory nerve endings. ${ }^{17}$ Because esophageal $\mathrm{BI}$ is negatively correlated with DIS, it may be related to the generation of certain symp- 

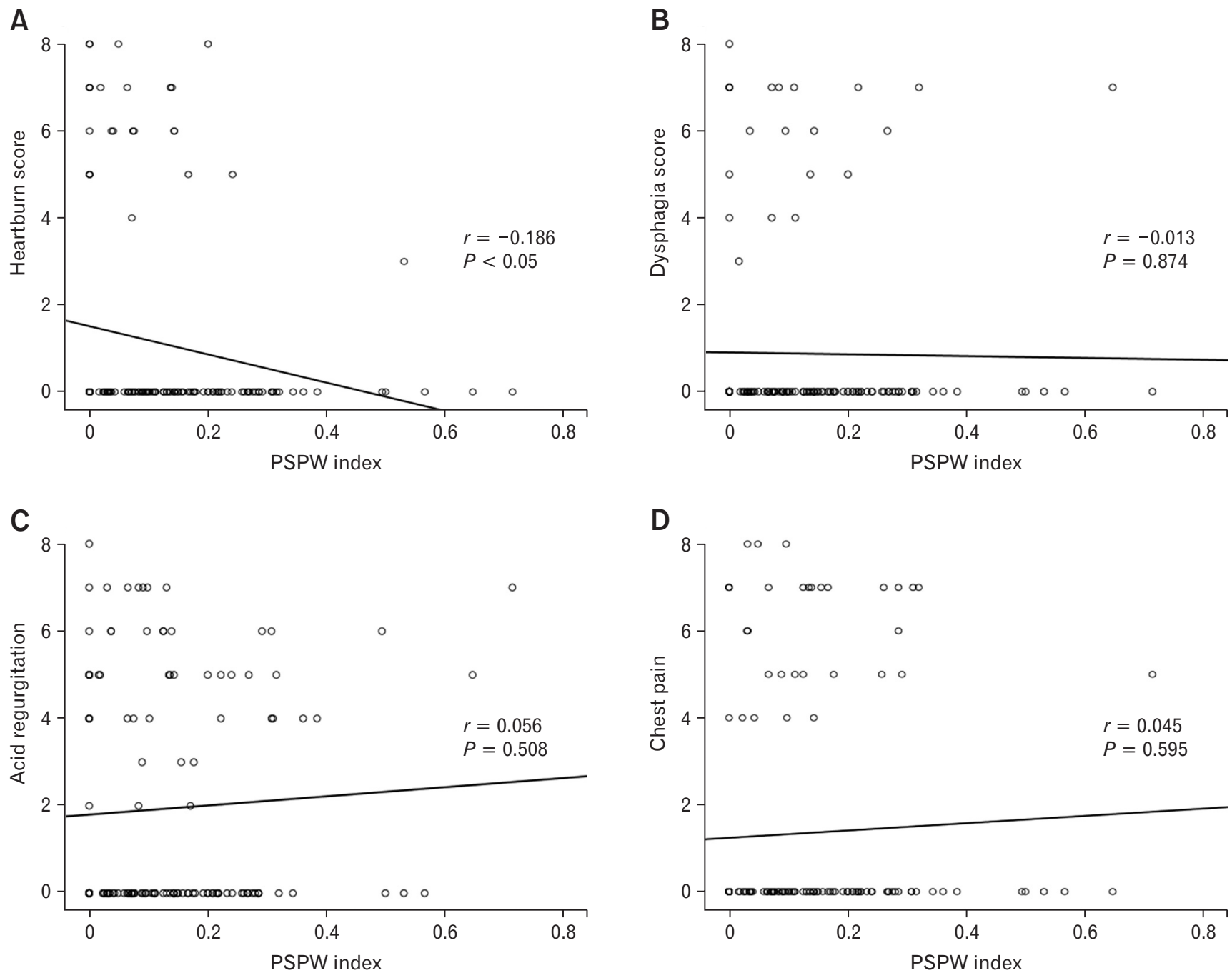

Figure 2. Relationship between post-reflux swallow-induced peristaltic wave (PSPW) and gastroesophageal reflux disease symptoms. (A) PSPW index negatively correlated with heartburn score $(r=-0.186, P<0.05)$. (B) PSPW index and dysphagia score was not significantly related $(r=$ $-0.013, P=0.874)$. (C) PSPW index and acid regurgitation was not significantly related $(r=0.056, P=0.508)$. (D) PSPW index and chest pain was not significantly related $(r=0.045, P=0.595)$.

Table 2. Classified Reflux Episodes According to Acidity, Post-reflux Swallow-induced Peristaltic Wave, and Neutralization

\begin{tabular}{lrr}
\hline \multicolumn{1}{c}{ Categories of reflux events } & Mean (SD) & Total number \\
\hline Acid reflux not followed by PSPW & $13.78(12.12)$ & 1970 \\
Acid reflux followed by PSPW with neutralization & $1.83(3.16)$ & 260 \\
Acid reflux followed by PSPW without neutralization & $1.05(2.75)$ & 148 \\
Non-acid reflux not followed by PSPW & $12.98(10.72)$ & 1843 \\
Non-acid reflux followed by PSPW & $1.40(2.70)$ & 199 \\
Total reflux & $30.91(19.35)$ & 4420 \\
Total PSPW & $4.24(6.35)$ & 607 \\
PSPW index & $0.13(0.14)$ & \\
\hline
\end{tabular}

PSPW, post-reflux swallow-induced peristaltic wave. 

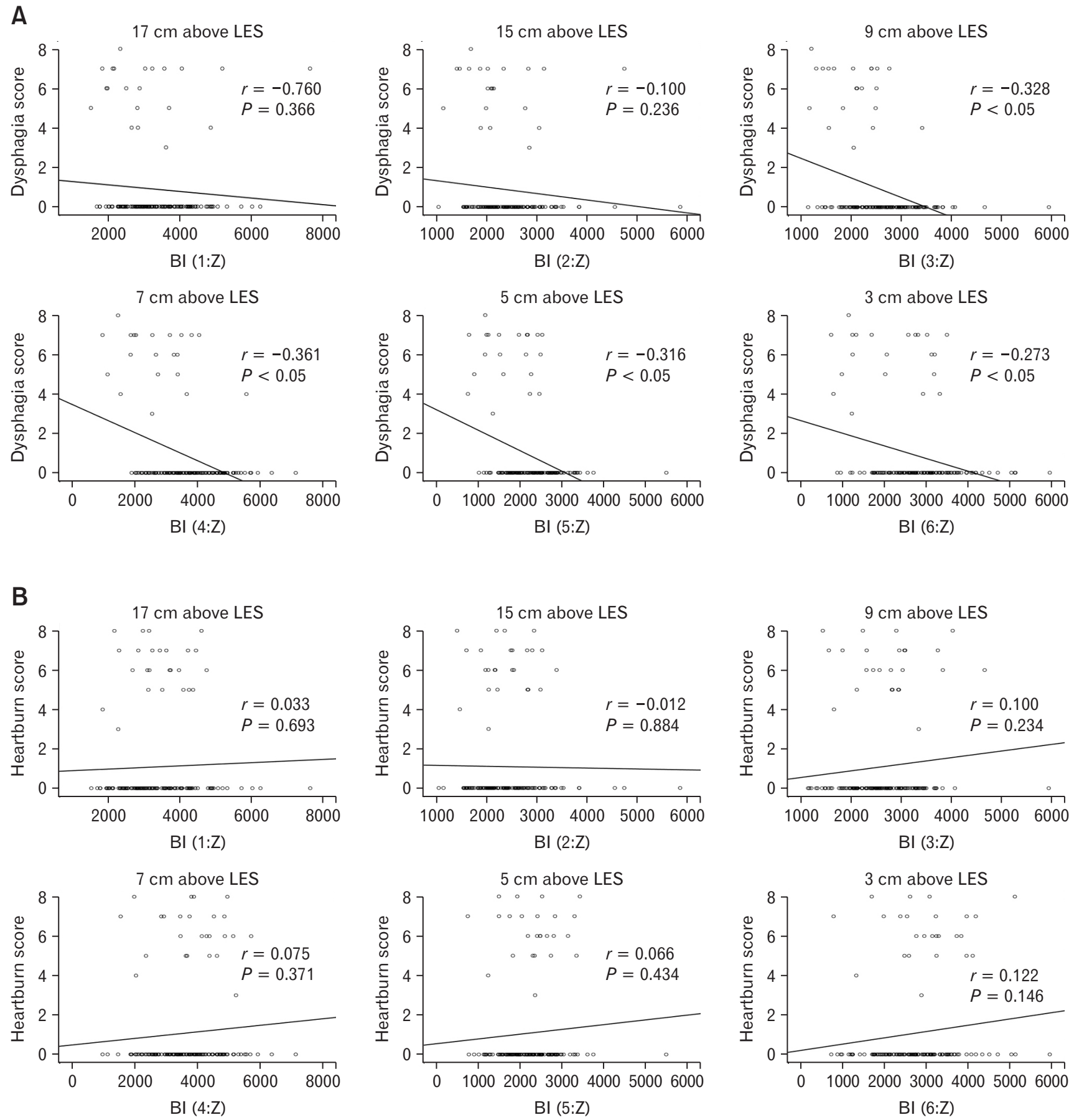

Figure 3. Relationships between esophageal baseline impedance and dysphagia and heartburn. (A) Negative association of distal esophageal baseline impedance $(\mathrm{BI}$; ohm) at $3 \mathrm{~cm}, 5 \mathrm{~cm}, 7 \mathrm{~cm}$, and $9 \mathrm{~cm}$ above lower esophageal sphincter (LES) and dysphagia score. Proximal esophageal baseline impedance levels at $15 \mathrm{~cm}$ and $17 \mathrm{~cm}$ above LES were not significantly related with dysphagia score. (B) Esophageal BI was not significantly correlated with heartburn score. 
toms. A well-known secondary defense mechanism against GERD is esophageal acid clearance, for which generation of peristalsis is very important. ${ }^{5}$ The PSPW index represents a direct function of chemical clearance. ${ }^{5}$ As the human esophageal mucosa is very sensitive to continuous exposure to acidic and weakly acidic solutions, ${ }^{18}$ a decreased PSPW index may underlie certain symptoms via decreased esophageal clearance or hypersensitivity of the esophagus.

However, the exact mechanisms underlying each GERD symptom are not obvious. In this study, we investigated the direct associations of GERD symptoms with the PSPW index and esophageal BI.

The PSPW index was inversely correlated with heartburn but was not significantly associated with other symptoms such as dysphagia, acid regurgitation, chest pain, cough, hoarseness, and globus sense. A previous study showed that the PSPW index was significantly lower in patients with ERD than in those with NERD and was lower in patients with NERD than in controls or patients with functional heartburn. ${ }^{5}$ These findings suggest that patients with ERD may have greater neuronal damage or neuritis that inhibits the initiation of esophageal peristalsis. Compared with NERD, in ERD, a clear breach in the squamous epithelium allows reflux components to reach acid-sensitive nerve endings, including acid-sensitive ion channels located in the mucosa and lamina propria, which deliver the sensations of heartburn and chest pain to the central nervous system. ${ }^{19,20}$ The PSPW index may be related to heartburn development; however, the exact underlying mechanism, such as acid sensitization due to a longer exposure to esophageal acid, remains to be elucidated.

In our study, the mean number of acid reflux events followed by PSPWs regardless of neutralization (2.8) was greater than the mean number of non-acid reflux events followed by PSPWs (1.4). This finding suggests that acid reflux is a more reliable factor triggering PSPWs than non-acid reflux. The PSPW index was not related to dysphagia in our study. The etiology of dysphagia in GERD patients is controversial. In a previous study, abnormal sensory perception in the esophagus led to the perception of dysphagia, even when the bolus had cleared the esophagus. ${ }^{21}$ Future studies are warranted to confirm these findings.

Esophageal BI, a measure of mucosal integrity was inversely correlated with intercellular space in a previous study. ${ }^{6}$ In our study, distal esophageal BI was inversely correlated with dysphagia but was not associated with heartburn. Other symptoms including acid regurgitation and chest pain showed weak and sporadic positive correlations with proximal esophageal BI. Another study showed that esophageal perfusion with acid solutions provoked DIS in the exposed esophageal mucosa. ${ }^{18}$ Interestingly, despite the presence of perfusion induced DIS, most healthy subjects did not perceive heartburn, thus casting doubt on the existence of a direct relationship between DIS and reflux symptoms. ${ }^{18}$ This phenomenon may be related to the peripheral sensitization in GERD patients. Reflux events inducing DIS and allowing peripheral sensitization of subepithelial nerves by gastric acid or other refluxed materials could contribute to the increased perception of esophageal sensations including dysphagia. ${ }^{22}$ Consequently, decreased esophageal BI may be related to increased dysphagia symptoms. Regarding the positive relationships between acid regurgitation/chest pain and proximal esophageal BI in our study, we could not find any reasonable relationship. However, the regurgitation may increase the gas content in the proximal esophagus which may increase the intraluminal impedance level at the segment of air trapping, because MII-pH catheter measures the intraluminal impedance rather than the mucosal impedance. Distended proximal esophagus by regurgitation and trapped gas may also induce chest pains. But, these findings were very weak correlations and sporadic. We should be cautious in interpreting these primitive observations. Further studies should be performed to determine the pathophysiological mechanisms underlying GERD symptoms.

A few limitations of our study should be acknowledged. First of all, it was a retrospective study. We analyzed Impedance-pH of patients who had GERD symptoms by medical records. Second, although the questionnaires and scoring system for the analysis of symptoms were developed, the symptoms can be subjective. Third, we did not classify the patients as reflux esophagitis, nonerosive reflux disease, and functional heartburn, because (1) our subjects did not undergo the PPI test because of the retrospective design and (2) the major limitation of Imp-pH is false negative. Fourth, although all subjects received endoscopy and high resolution manometry, we could not precisely exclude early phases of systemic sclerosis and eosinophilic esophagitis because we performed esophageal mucosal biopsy in only suspected patients (not routinely performed). All of the above are limitations of a retrospective study. Even though there are some limitations, this study is meaningful in the respect that GERD symptoms with PSPW and BI were directly compared. Classification of reflux episodes according to acidity, PSPW and neutralization was the first attempt to date regardless of the results.

In conclusion, we found that chemical clearance of the secondary defense mechanism, measured using the PSPW index, was inversely correlated with heartburn but not dysphagia. Moreover distal esophageal mucosal integrity of the tertiary defense mechanism, measured using esophageal BI, was inversely correlated with 
dysphagia but not heartburn. These correlations were weak, thus, future studies should aim to elucidate the underlying pathophysiological mechanisms of GERD symptoms and their associations with the PSPW index and esophageal BI.

\section{Supplementary Materials}

Note: To access the supplementary figures mentioned in this article, visit the online version of Journal of Neurogastroenterology and Motility at http://www.jnmjournal.org/, and at https://doi. org/10.5056/jnm16115.

Acknowledgements: We would like to express our sincere gratitude to Yun Jung, study codinator of the department of Gastroenterology, Soonchunhyang University who helped to acquire patients' data of impedance $\mathrm{pH}$ monitoring. The manuscript has been presented at last Digestive Disease Week (DDW) 2016 in San Diego, California.

Financial Support: This work was supported in part by the Soonchunhyang University Research Fund.

\section{Conflicts of interest: None.}

Author contributions: Young Kyu Cho has contributed in data collection, analysing of data, and writing as first author; Joon Seong Lee has contributed in study design, editing the paper and supervised this study as corresponding author; Tae Hee Lee and Su Jin Hong have contributed in data acquisition; and Sang Joon Park, Seong Ran Jeon, Hyun Gun Kim, and Jin-Oh Kim have contributed in review and editing the manuscript

\section{References}

1. Kahrilas PJ, Shaheen NJ, Vaezi MF, et al. American Gastroenterological Association Medical Position Statement on the management of gastroesophageal reflux disease. Gastroenterology 2008;135:1383-1391, e1-e5.

2. Mouli VP, Ahuja V. Questionnaire based gastroesophageal reflux disease (GERD) assessment scales. Indian J Gastroenterol 2011;30:108-117.

3. Blondeau K, Tack J. Pro: impedance testing is useful in the management of GERD. Am J Gastroenterol 2009;104:2664-2666.

4. Helm JF, Dodds WJ, Pelc LR, Palmer DW, Hogan WJ, Teeter BC. Effect of esophageal emptying and saliva on clearance of acid from the esophagus. N Engl J Med 1984;310:284-288.

5. Frazzoni M, Manta R, Mirante VG, Conigliaro R, Frazzoni L, Melotti G. Esophageal chemical clearance is impaired in gastro-esophageal reflux disease-a 24-h impedance-pH monitoring assessment. Neurogastroenterol Motil 2013;25:399-406.
6. Zhong C, Duan L, Wang K, et al. Esophageal intraluminal baseline impedance is associated with severity of acid reflux and epithelial structural abnormalities in patients with gastroesophageal reflux disease. J Gastroenterol 2013;48:601-610.

7. Farré R, Blondeau K, Clement D, et al. Evaluation of oesophageal mucosa integrity by the intraluminal impedance technique. Gut 2011;60:885892.

8. Orlando LA, Orlando RC. Dilated intercellular spaces as a marker of GERD. Current Gastroenterology Reports 2009;11:190-194.

9. van Malenstein H, Farré R, Sifrim D. Esophageal dilated intercellular spaces (DIS) and nonerosive reflux disease. Am J Gastroenterol 2008;103:1021-1028.

10. Tutuian R, Vela MF, Balaji NS, et al. Esophageal function testing with combined multichannel intraluminal impedance and manometry: multicenter study in healthy volunteers. Clin Gastroenterol Hepatol 2003;1:174182.

11. Bredenoord AJ, Weusten BL, Timmer R, Smout AJ. Reproducibility of multichannel intraluminal electrical impedance monitoring of gastroesophageal reflux. Am J Gastroenterol 2005;100:265-269.

12. Shafik A, El-Sibai O, Shafik AA, Mostafa R. Effect of topical esophageal acidification on salivary secretion: identification of the mechanism of action. J Gastroenterol Hepatol 2005;20:1935-1939.

13. Ang D, Sifrim D, Tack J. Mechanisms of heartburn. Nat Clin Pract Gastroenterol Hepatol 2008;5:383-392.

14. Rodriguez-Stanley S, Robinson M, Earnest DL, Greenwood-Van Meerveld B, Miner PB Jr. Esophageal hypersensitivity may be a major cause of heartburn. Am J Gastroenterol 1999;94:628-631.

15. Barish CF, Castell DO, Richter JE. Graded esophageal balloon distension. A new provocative test for noncardiac chest pain. Dig Dis Sci 1986;31:1292-1298.

16. Sifrim D, Mittal R, Fass R, et al. Review article: acidity and volume of the refluxate in the genesis of gastro-oesophageal reflux disease symptoms. Aliment Pharmacol Ther 2007;25:1003-1017.

17. Caviglia R, Ribolsi M, Gentile M, et al. Dilated intercellular spaces and acid reflux at the distal and proximal oesophagus in patients with nonerosive gastro-oesophageal reflux disease. Aliment Pharmacol Ther 2007;25:629-636.

18. Farré R, Fornari F, Blondeau K, et al. Acid and weakly acidic solutions impair mucosal integrity of distal exposed and proximal non-exposed human oesophagus. Gut 2010;59:164-169.

19. Weijenborg PW, Bredenoord AJ. How reflux causes symptoms: reflux perception in gastroesophageal reflux disease. Best Pract Res Clin Gastroenterol 2013;27:353-364.

20. Holzer P. Acid-sensing ion channels in gastrointestinal function. Neuropharmacology 2015;94:72-79.

21. Clouse RE, McCord GS, Lustman PJ, Edmundowicz SA. Clinical correlates of abnormal sensitivity to intraesophageal balloon distension. Dig Dis Sci 1991;36:1040-1045.

22. Szczesniak MM, Fuentealba SE, Cook IJ. Acid sensitization of esophageal mucosal afferents: implication for symptom perception in patients across the gastroesophageal reflux disease spectrum. Clin J Pain 2013;29:70-77. 